\title{
Volume effects on fatigue life of equine cortical bone
}

\author{
R.F. Bigley ${ }^{\mathrm{a}, *, 1}$, J.C. Gibeling ${ }^{\mathrm{b}}$, S.M. Stover ${ }^{\mathrm{c}}$, S.J. Hazelwood ${ }^{\mathrm{a}}$, D.P. Fyhrie ${ }^{\mathrm{a}}$, R.B. Martin ${ }^{\mathrm{a}}$ \\ ${ }^{a}$ Orthopaedic Research Laboratories, School of Medicine, UC Davis Medical Center, 4635 Second Avenue, Sacramento, CA 95817 \\ ${ }^{\mathrm{b}}$ Department of Chemical Engineering and Materials Science, College of Engineering \\ ${ }^{\mathrm{c}}$ J.D. Wheat Veterinary Orthopedic Research Laboratory, School of Veterinary Medicine, University of California at Davis, Davis, CA 95616
}

\begin{abstract}
Materials, including bone, often fail due to loading in the presence of critical flaws. The relative amount, location, and interaction of these flaws within a stressed volume of material play a role in determining the failure properties of the structure. As materials are generally imperfect, larger volumes of material have higher probabilities of containing a flaw of critical size than do smaller volumes. Thus, larger volumes tend to fail at fewer cycles compared with smaller volumes when fatigue loaded to similar stress levels. A material is said to exhibit a volume effect if its failure properties are dependent on the specimen volume. Volume effects are well documented in brittle ceramics and composites and have been proposed for bone. We hypothesized that (1) smaller volumes of cortical bone have longer fatigue lives than similarly loaded larger volumes and (2) that compared with microstructural features, specimen volume was able to explain comparable amounts of variability in fatigue life. In this investigation, waisted rectangular specimens $(n=48)$ with nominal cross-sections of $3 \times 4 \mathrm{~mm}$ and gage lengths of $10.5,21$, or $42 \mathrm{~mm}$, were isolated from the mid-diaphysis of the dorsal region of equine third metacarpal bones. These specimens were subjected to uniaxial load controlled fatigue tests, with an initial strain range of 4000 microstrain. The group having the smallest volume exhibited a trend of greater log fatigue life than the larger volume groups. Each volume group exhibited a significant positive correlation between the logarithm of fatigue life and the cumulative failure probability, indicating that the data follow the two-parameter Weibull distribution. Additionally, log fatigue life was negatively correlated with log volume, supporting the hypothesis that smaller stressed volumes of cortical bone possess longer fatigue lives than similarly tested larger stressed volumes.
\end{abstract}

Keywords: Equine third metacarpal; Fatigue life; Weibull analysis; Volume; Failure

\section{Introduction}

Cortical bone is analogous to a fiber-reinforced ceramic matrix composite with the osteons acting as fibers within an interstitial matrix (Currey, 1964; Hogan, 1992; Buckwalter et al., 1995; Martin et al., 1998; Bigley et al., 2006). Ceramic matrix composites often show fatigue life variation due to the number and distribution of defects (Weibull, 1951; Hertzberg, 1996; Wisnom, 1999; Cattell and Kibble, 2001; Rentzsch, 2003). Although osteonal bone possesses several advantageous properties associated with ceramic matrix composites, it also possesses defect populations. Microcracks, regions of poor collagen quality, and microstructural components acting as stress concentrators (e.g. osteons, Haversian canals, resorption cavities) have been associated with low toughness in cortical bone (Schaffler et al., 1995; Wang and Puram, 2004; O'Brien et al., 2005). These interactions of microcracks with the microstructural components suggest that a single value of material strength or toughness is not sufficient to characterize the failure behavior of bone tissue.

\subsection{Weibull statistical theory}

Brittle material failure can be caused by a single critical defect or by several small defects acting together to create a critical flaw (Wisnom, 1999). The Weibull "weakest link" approach enables failure characterization when defects can 
be assumed to be randomly distributed throughout a material (Weibull, 1951). Due to the statistical nature of the occurrence and size of such defects in ceramic materials, many exhibit a volume effect. A material is said to exhibit a volume effect if the probability of failure increases in proportion to the stressed volume of the specimen. Griffith (1920) reported that failure strength increased with decreased fiber diameter in his pioneering work on brittle solids. Such volume effects are fundamental to understanding failure probability (Wisnom, 1999; Rentzsch, 2003).

If failure is determined by the presence of a critical defect, and such defects occur randomly within a material, it follows that for a given stress, larger volumes of a material will have higher failure probabilities because they have a higher probability of possessing a critical defect (Hertzberg, 1996). Weibull's statistical theory can be used to characterize the variability in fatigue life associated with the size of a structure or test specimen (Wisnom, 1999; Cattell and Kibble, 2001).

The two-parameter Weibull fatigue life model can be used to describe the failure probability, $P$, of a volume, $V$, of a material subject to a cyclically applied uniform stress field:

$P=4-\exp \left[V\left(\frac{N_{\mathrm{f}}}{N_{0}}\right)^{m_{\mathrm{f}}}\right]$

where $N_{\mathrm{f}}$ is the number of load cycles, $N_{0}$ is the characteristic fatigue life of a unit volume, and $m_{\mathrm{f}}$ is the Weibull fatigue modulus (Weibull, 1951; Hertzberg, 1996; Wisnom, 1999; Cattell and Kibble, 2001). The fatigue modulus describes the shape of the distribution and is representative of the fatigue life variability among test specimens of the same volume tested under similar stress conditions. High values of $m_{\mathrm{f}}$ correspond to reduced variability of fatigue life, while small values of $m_{\mathrm{f}}$ are associated with increased variability in fatigue life (Rentzsch, 2003).

\subsection{Volume effects in bone}

Taylor (1998) analyzed fatigue strength data from numerous sources and published a model for bone fatigue strength based on stressed volume. In this volume effects model, the fatigue strength of human bone was defined as the failure stress range in $\mathrm{MPa}$, determined from a stresslife curve, for a fatigue life of 100,000 cycles for bone tested wet at $2 \mathrm{~Hz}$ in zero-to-tension corrected to $37^{\circ} \mathrm{C}$ (Taylor, 1998; Taylor et al., 1999). The study demonstrated that prediction of bone fatigue behavior from small test specimens is not applicable to larger specimens unless the volume effect is considered (Taylor, 1998). Taylor and Kuiper later combined this stressed volume model with finite element modeling to predict clinical stress fracture probabilities in the human tibia (Taylor and Kuiper, 2001). The model was later refined to incorporate the effects of remodeling and damage (Taylor et al., 2004). Weibull theory has also been used to analyze equine and human fatigue data to investigate the implications of volume effects on bone adaptation (Yeh and Martin, 2003). This investigation revealed Weibull fatigue life moduli ranging between 0.5 and 1.5 for initial strain amplitudes between -5000 and $+10,000$ microstrain. Although these studies successfully used the Weibull distribution to analyze fatigue strength data, to our knowledge no a priori study has directly tested the applicability of the Weibull distribution to cortical bone strengths measured using specimens of different volumes.

We hypothesize that strength volume effects in cortical bone are manifested in fatigue life and can be characterized by Weibull theory. Consequently, for a given applied stress, smaller stressed volumes have a lower probability of failure and will thus possess a statistically longer fatigue life when compared with larger stressed volumes. Additionally, we hypothesized that the observed volume effect explains variability of the fatigue life of equine cortical bone not accounted for by microstructural variables.

\section{Methods}

\subsection{Experimental preparation}

Originally, 30 specimens were obtained from the third metacarpal (cannon) bones of 15 necropsied Thoroughbred racehorses. These racehorses consisted of 4 females, 3 males, and 8 castrated males with ages ranging between 2 and 7 years. The bone specimens were kept frozen at $-20^{\circ} \mathrm{C}$ except while machining or testing, when they were hydrated with saline at room temperature. One rectangular beam, nominally $140 \times 15 \times 6 \mathrm{~mm}$, was cut with a bone saw (Hobart Corp, Troy, OH) from the dorsal region of the mid-diaphysis from each bone. The beam's long axis was aligned with the anatomical long axis of the bone. The width $(15 \mathrm{~mm})$ and thickness $(6 \mathrm{~mm})$ corresponded to the circumferential and radial anatomical directions, respectively.

Using a computer numeric controlled (CNC) mill (Prolight 1000, Light Machines Corporation, Manchester, NH) specimens were wet machined into three different volume groups. The gage lengths of the rectangular waisted tensile test specimens resulted in volumes of 126,252 , or $504 \mathrm{~mm}^{3}$ (Fig. 1). The machined specimens were then lightly polished with 800 -grit carbide paper to remove any surface artifacts from the machining process.

The machined specimens were tested in random order. Each specimen was thermally equilibrated for $30 \mathrm{~min}$, and fatigue tested under constant irrigation with calcium-buffered saline solution at $37^{\circ} \mathrm{C}$ (Gustafson et al., 1996). Fatigue testing was performed using an MTS 810 servohydraulic testing machine (MTS Corporation, Eden Prairie, MN) running Testware SX software in accordance with the guidelines specified in the American Society for Testing and Materials Standard E8M-01. Sinusoidal $(2 \mathrm{~Hz})$ fatigue tests to failure were conducted in load control using a predetermined initial strain range of $\Delta \varepsilon_{i}=4000 \mu \varepsilon$. This initial strain range was selected based on previously reported strains for Thoroughbred racehorses (Nunamaker et al., 1990; Gibson et al., 1995). Strain was measured by an extensometer (MTS models 632.26E-30, 632.26B-30, 632.12B-30) attached with small elastic bands to the waisted region. Load was measured using a $2446 \mathrm{~N}(550 \mathrm{lb})$ capacity load cell (MTS model 661.18C-02).

Each specimen underwent 100 preconditioning cycles $(10-100 \mathrm{~N})$ using a $2 \mathrm{~Hz}$ sinusoidal waveform. The tensile elastic modulus of each test specimen was then defined as the average modulus of the last 3 cycles of a 6-cycle triangular waveform, applied at $2 \mathrm{~Hz}$, for a loading range of $10-600$ N. Subsequently, the force, $F$, necessary to produce the desired initial fatigue test strain, $\varepsilon_{i}=4000 \mu \varepsilon$, was calculated from the elastic 
modulus, $E$, and the cross-sectional area of the test specimen, $A$, using

$F=\leftarrow_{l} A E$.

The fatigue life tests were conducted using a $2 \mathrm{~Hz}$ sinusoidal waveform between $10 \mathrm{~N}$ and the calculated force, $F$. Failure was defined as complete fracture of the test specimen. The number of cycles to reach failure, $N_{\mathrm{f}}$, for each test specimen was recorded as the fatigue life. Specimens were excluded from further analysis if failure occurred outside the waisted gage region.

\subsection{Analysis of variance}

The effect of volume $\left(V_{1}, V_{2}, V_{3}\right)$ on $\log \left(N_{\mathrm{f}}\right)$ was assessed using a mixed model analysis of variance that accounted for repeated measures within horses, and included age as a continuous variable and sex as a categorical (female, male, castrated male) fixed effect. The horse-subject effect was treated as either a fixed or random effect in separate models. Least-squares means adjusted for other factors in the model were used to assess pairwise comparisons (SAS v9.1.3, Cary, NC). Statistical significance was reported for $p<0.05$.

\subsection{Individual Weibull analyses}

The Weibull modulus, $m_{\mathrm{f}}$, and characteristic fatigue life, $N_{0}$, were determined for the three different volume groups based on their respective failure probabilities. Within each group, fatigue life was ranked from shortest to longest and the respective failure probabilities were calculated using the Bernard median rank (Wisnom, 1999; Cattell and Kibble, 2001). Median rank is an estimator of the true failure probability, $P_{i}$, for the $i$ th ranked fatigue life, $N_{i}$, and is given by

$P_{i}=\frac{i-0.3}{n+0.4}$

where $i$ is the rank of the fatigue life and $n$ is the group sample size ( $n=6$ for each volume group) (Cattell and Kibble, 2001). For each volume group, Eq. (1) can be rewritten in linear form by taking the natural logarithm twice,

$\ln \ln \left(\frac{1}{1-P_{i}}\right)=4_{\mathrm{f}} \ln \left(N_{i}\right)-m_{\mathrm{f}} \ln \left(N_{0}\right)$,

where $P_{i}$ is the failure probability, $m_{\mathrm{f}}$ is the Weibull fatigue modulus, and $N_{0}$ is the characteristic fatigue life. Regression of the failure probability against the fatigue life allows each volume's fatigue modulus and characteristic fatigue life to be calculated (SAS v9.1.3, Cary, NC).

\subsection{Combined volume effects analysis}

Assuming equal material failure probabilities, the volume effect on fatigue life ratio for two specimens of volumes $V_{\mathrm{A}}$ and $V_{\mathrm{B}}$ is

$\frac{N_{\mathrm{A}}}{N_{\mathrm{B}}}=\left(\frac{V_{\mathrm{B}}}{V_{\mathrm{A}}}\right)^{1 / m_{\mathrm{v}}}$,

where $N_{\mathrm{A}}$ and $N_{\mathrm{B}}$ are the respective fatigue lives, and $m_{\mathrm{V}}$ is the Weibull volume modulus (Wisnom, 1999; Cattell and Kibble, 2001). For geometrically similar specimens with uniform stress distributions, the volume effect on fatigue life can be determined from a log-log plot of Eq. (5), where the slope is the negative reciprocal of the Weibull volume modulus, $m_{\mathrm{V}}$ (Wisnom, 1999; Cattell and Kibble, 2001). The distinction between fatigue modulus, $m_{\mathrm{f}}$, and volume modulus, $m_{\mathrm{V}}$, is in the manner of determination. The Weibull fatigue modulus is determined within individual volume groups while the Weibull volume modulus is determined across the different volume groups. For a material that can be described by the Weibull distribution, the two values should be similar because they are derived from the same distribution (Wisnom, 1999; Cattell and Kibble, 2001).

\subsection{Histomorphometry}

Transverse sections of the specimens were cut just proximal and distal to the fracture surface of each fragment using a low-speed diamond saw (Isomet Buehler, Lake Bluff, IL). These sections (100 $\pm 10 \mu \mathrm{m}$ thick) were subsequently mounted onto glass slides using Eukitt mounting media (Calibrated Instruments, Hawthorne, NY) and underwent histomorphometric analysis.

The histomorphometric analysis was conducted over the entire crosssection (nominally $3 \times 4 \mathrm{~mm}$ ) on both the proximal and distal sections from each specimen. Images of the cross-section were acquired using an Olympus BH2 microscope and CCD camera with an objective magnification of $10 \mathrm{X}$. A $3 \times 3$ grid, totaling nine images, spanned the entire crosssection. This analysis area was defined as B.Ar $=2.6 \times 3.2 \mathrm{~mm}=\leftarrow$ $8.32 \mathrm{~mm}^{2}$

The equine specimens were highly remodeled, leaving very few primary osteons. Secondary osteons were identified and counted by the presence of a prominent cement line. Additionally, secondary osteons were counted as complete if they possessed three radii covering greater than $80 \%$ of the osteonal area. The number of osteons in each analysis area was counted and divided by the analysis area, B.Ar, to obtain the osteon density (On.Dn, \#/ $\mathrm{mm}^{2}$ ):

On.Dn $=\frac{\text { N.On }}{\text { B.Ar }}$

Two perpendicular cement line diameters were measured and averaged for six randomly selected osteons in each of the nine fields, resulting in a total of 54 osteon diameters per section. The osteon diameter (On.Dm, $\mathrm{mm}$ ) for each specimen was defined as the average of the cement line diameters $(54$ diameters/section $\times 2$ sections/specimen $=408$ diameters $)$ for each specimen.

Haversian canal diameters (H.Dm, mm) were measured in the same manner as cement line diameter, for each of the six randomly selected osteons. Porous cavities (resorption cavities and Volkmann's canals) were counted (N.Po) and measured using two perpendicular diameters (Po.Dm, $\mathrm{mm}$ ) across each of the nine fields. Total porosity (PO, \%) was defined as the combined Haversian canal area and porous cavity area divided by the total analysis area (B.Ar):

$\mathrm{PO}=\frac{(\pi / 4)\left[\mathrm{N} \cdot \mathrm{Po}\left(\mathrm{Po} \cdot \mathrm{Dm}^{2}\right)+\mathrm{N} \cdot \mathrm{On}\left(\mathrm{H} \cdot \mathrm{Dm}^{2}\right)\right]}{\text { B.Ar }} \leftarrow$

Regression analyses (SAS v9.1.3, Cary, NC) were performed to determine whether histomorphometric parameters correlated with specimen volume or the measured mechanical properties, including elastic modulus, and fatigue life. Statistical significance was reported for $p<0.05$.

\section{Results}

Twelve test specimens were excluded from the analysis because failure occurred outside of the waisted gage region. The current analysis consists of 18 test specimens from 14 racehorses ( 3 females, 3 males, 8 castrated males) between 2 and 6 years.

\subsection{Analysis of variance}

The mixed model repeated measures analysis of variance with horse as a repeated random subject resulted in nearly significant volume $(p=\theta .071)$ differences in $\log \left(N_{\mathrm{f}}\right)$, while age $(p=\theta .380)$ and sex $(p=\$ .276)$ did not demonstrate significant effects on $\log \left(N_{\mathrm{f}}\right)$. Subsequent post hoc comparisons showed that the largest volume group, $V_{3}$, had a significantly lower mean $\log \left(N_{\mathrm{f}}\right)$ than the intermediate 
Table 1

Summary of various physical, mechanical, and histomorphometric parameters for the 18 equine specimens (mean \pm standard deviation)

\begin{tabular}{|c|c|c|c|}
\hline \multirow[t]{2}{*}{ Parameter } & \multicolumn{3}{|c|}{ Specimen volume group } \\
\hline & $V_{1}$ & $V_{2}$ & $V_{3}$ \\
\hline Age (years) & $4.0 \pm 0.6$ & $4.7 \pm 0.5$ & $3.5 \pm 1.6$ \\
\hline Volume, $V\left(\mathrm{~mm}^{3}\right)$ & $118.3 \pm 11.6$ & $248.8 \pm 4.9$ & $497.5 \pm 7.4$ \\
\hline Fatigue life, $N_{\mathrm{f}}$ (cycles) & $17,392 \pm 11,184$ & $14,511 \pm 9565$ & $6741 \pm 3919$ \\
\hline $\log \left(N_{\mathrm{f}}\right)$ & $4.17 \pm 0.27$ & $4.09 \pm 0.28$ & $3.75 \pm 0.32$ \\
\hline Weibull fatigue modulus, $m_{\mathrm{f}}$ & 1.65 & 1.62 & 1.43 \\
\hline $95 \%$ Confidence interval $\left(m_{\mathrm{f}}\right)$ & $(0.95,2.36)$ & $(1.17,2.07)$ & $(1.06,1.81)$ \\
\hline Characteristic fatigue life $N_{0}$, (cycles) & 20,055 & 16,677 & 7927 \\
\hline Osteon diameter, On.Dm $(\mu \mathrm{m})$ & $167 \pm 33$ & $161 \pm 20$ & $162 \pm 11$ \\
\hline Porosity, PO (\%) & $4.3 \pm 2.6$ & $3.1 \pm 1.5$ & $2.9 \pm 1.2$ \\
\hline Osteon density, On.Dn ( $\left.\# / \mathrm{mm}^{2}\right)$ & $26.9 \pm 6.8$ & $24.9 \pm 4.7$ & $23.1 \pm 4.1$ \\
\hline
\end{tabular}

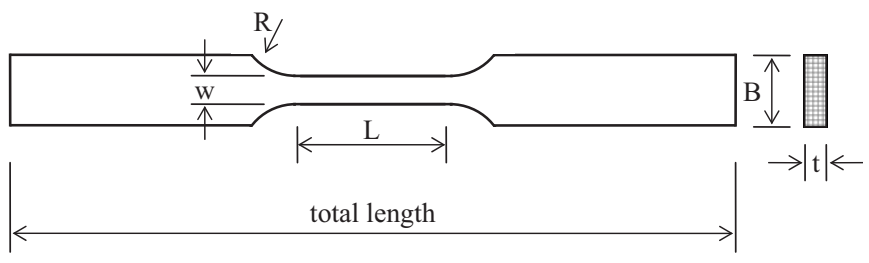

Fig. 1. Diagram of a tensile test specimen. All specimens had nominal dimensions of total length $=400 \mathrm{~mm}, R=4.875 \mathrm{~mm}, w=4 \mathrm{~mm}$, $B=40 \mathrm{~mm}$, and $t=3 \mathrm{~mm}$. Length was variable at $L=40.5,21$, and $42 \mathrm{~mm}$.

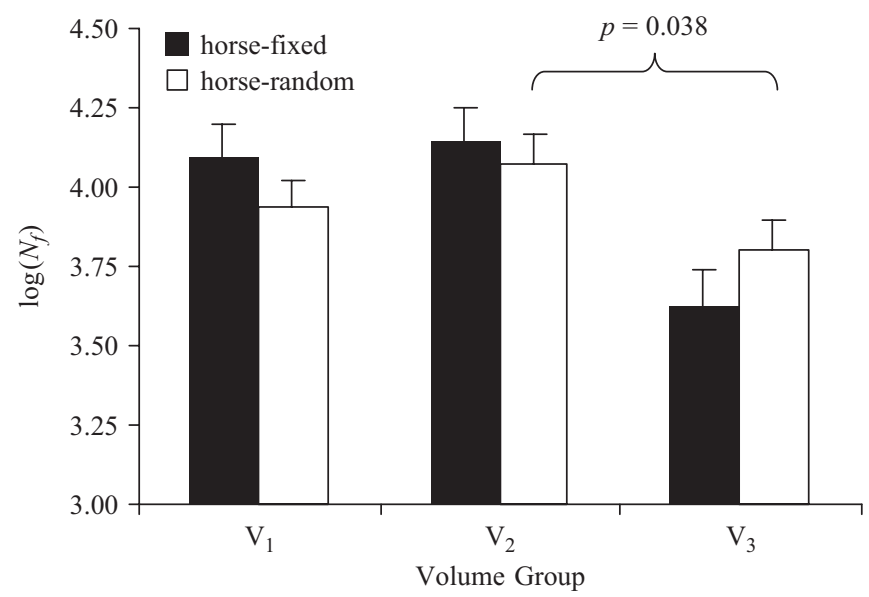

Fig. 2. Least squares mean log fatigue life for each volume group adjusted for age, sex and horse effects (mean \pm SD). In the fixed effects model, the largest volume group, $V_{3}$, had a nearly significant lower mean $\log \left(N_{\mathrm{f}}\right)$ than both the intermediate volume group, $V_{2}(p=\theta .089)$, and the small volume group, $V_{1}(p=\theta .083)$.

volume group, $V_{2}(p=\theta .038)$. Differences between volume groups $V_{1}$ and $V_{2}(p=0.090)$ and $V_{1}$ and $V_{3}(p=\theta .135)$ approached but did not reach significance. Similar trends existed when horse was treated as a fixed subject effect. Fatigue life and volume data are summarized in Table 1, while Fig. 2 presents $\log \left(N_{\mathrm{f}}\right)$ across the groups $V_{1}$, $V_{2}$, and $V_{3}$.

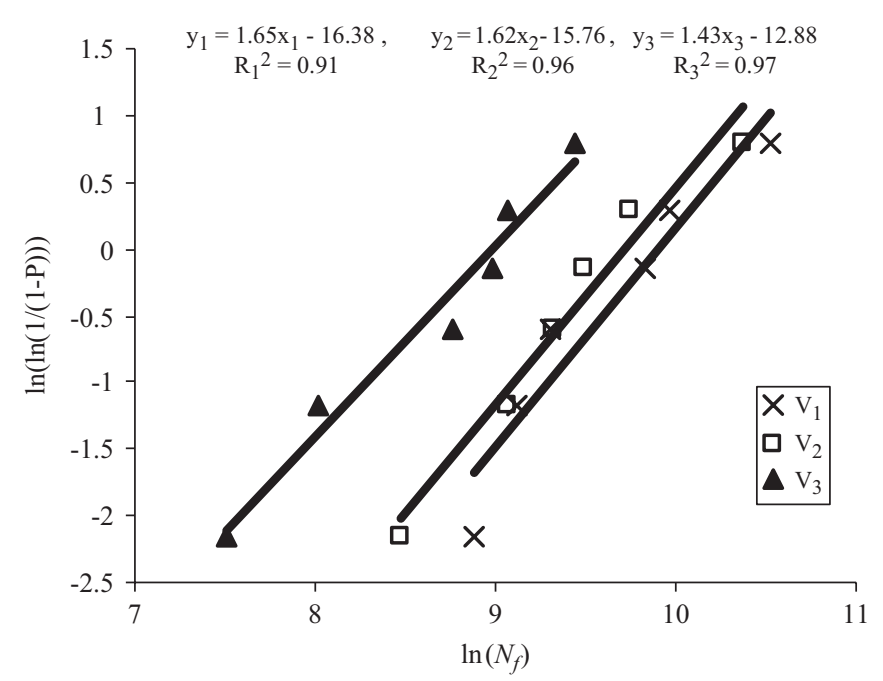

Fig. 3. Weibull probability plot for each volume group. Slopes are the Weibull fatigue moduli, $m_{\mathrm{f}}$. Intercepts on $\log \left(N_{\mathrm{f}}\right)$ axis are used to determine $N_{0}$.

\subsection{Individual Weibull analyses}

All three volume groups, $V_{1}, V_{2}$, and $V_{3}$, had significant regressions of the failure probability $(p)$ against $\log \left(N_{\mathrm{f}}\right)$ $(p=0.003, p=0.001, p<0.001$, respectively, Fig. 3.), demonstrating that the two-parameter Weibull model is applicable for the analysis. Individual Weibull fatigue moduli, $m_{\mathrm{f}}$, and characteristic fatigue lives, $N_{0}$, were calculated for each group (Eq. (4), Table 1). As expected, the Weibull fatigue moduli were not different (ANOVA, $p=0.656$ ) between the volume groups because the slopes are related to the specimen's material failure properties.

\subsection{Combined volume effects analysis}

Transformed fatigue life, $\log \left(N_{\mathrm{f}}\right)$, was significantly dependent on $\log (V)$ for all specimens (Fig. 4, linear regression, $\left.p=\theta .0187, R^{2}=\theta .30\right)$. The correlation was negative, demonstrating that fatigue life decreases with 
increases in volume. As expected, the Weibull volume modulus of $m_{\mathrm{V}}=4.47$ fell within the $95 \%$ confidence intervals of fatigue moduli $\left(m_{\mathrm{f}}\right)$ obtained from the individual analyses (Table 1) (Cattell and Kibble, 2001).

\subsection{Histomorphometry}

The summarized histomorphometric parameters for the fatigue data set are presented in Table 1. As expected, no significant correlations were detected between specimen volume measures and the histomorphometric parameters. Regression analyses between the histomorphometric parameters and the mechanical properties of elastic modulus, $E$, and the $\operatorname{logarithm}$ of fatigue $\operatorname{life}, \log \left(N_{\mathrm{f}}\right)$ yielded three significant results (Table 2). Porosity was negatively correlated with elastic modulus $\left(p<0.001, R^{2}=0.54\right.$, Fig. 5), but had no significant correlation with $\log \left(N_{\mathrm{f}}\right)$ ( $\left.p=\theta .652, R^{2}=\theta .01\right)$. Similarly, osteon density was also negatively correlated with elastic modulus $(p=\theta .033$, $R^{2}=\theta .25$, Fig. 6) and had no significant correlation with $\log \left(N_{\mathrm{f}}\right)\left(p=\theta .524, R^{2}=8.03\right)$. There was no significant correlation between osteon diameter and either elastic

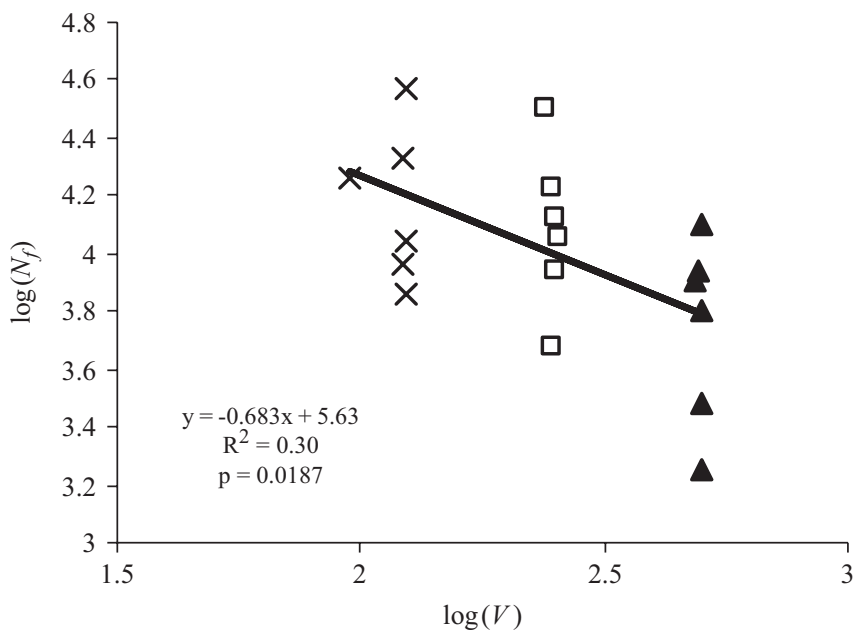

Fig. 4. Volume effects on fatigue life. One specimen in the small volume group $V_{1}$ had a smaller thickness than the others, causing it to have a smaller volume.

Table 2

Summary of regression analyses between specimen volume and histomorphometric parameters for the 18 fatigue specimens

\begin{tabular}{|c|c|c|c|c|c|c|}
\hline \multirow[t]{2}{*}{ Regression parameters } & \multicolumn{2}{|c|}{ Volume, $V$} & \multicolumn{2}{|c|}{ Elastic modulus, $E$} & \multicolumn{2}{|c|}{$\log \left(N_{\mathrm{f}}\right)$} \\
\hline & $p$ & $R^{2}$ & $p$ & $R^{2}$ & $p$ & $R^{2}$ \\
\hline Osteon size, On.Dm & 0.630 & 0.02 & 0.327 & 0.06 & 0.282 & 0.07 \\
\hline Porosity fraction, $\mathrm{PO}$ & 0.272 & 0.08 & 0.001 & 0.54 & 0.652 & 0.01 \\
\hline Osteon density, On.Dn & 0.270 & 0.08 & 0.033 & 0.25 & 0.524 & 0.03 \\
\hline Volume, $V$ & - & - & 0.336 & 0.06 & 0.012 & 0.33 \\
\hline Elastic modulus, $E$ & - & - & - & - & 0.587 & 0.02 \\
\hline
\end{tabular}

Bold values indicate statistically significant $(p<0.05)$ correlations between the histomorphometric parameter and the mechanical property.

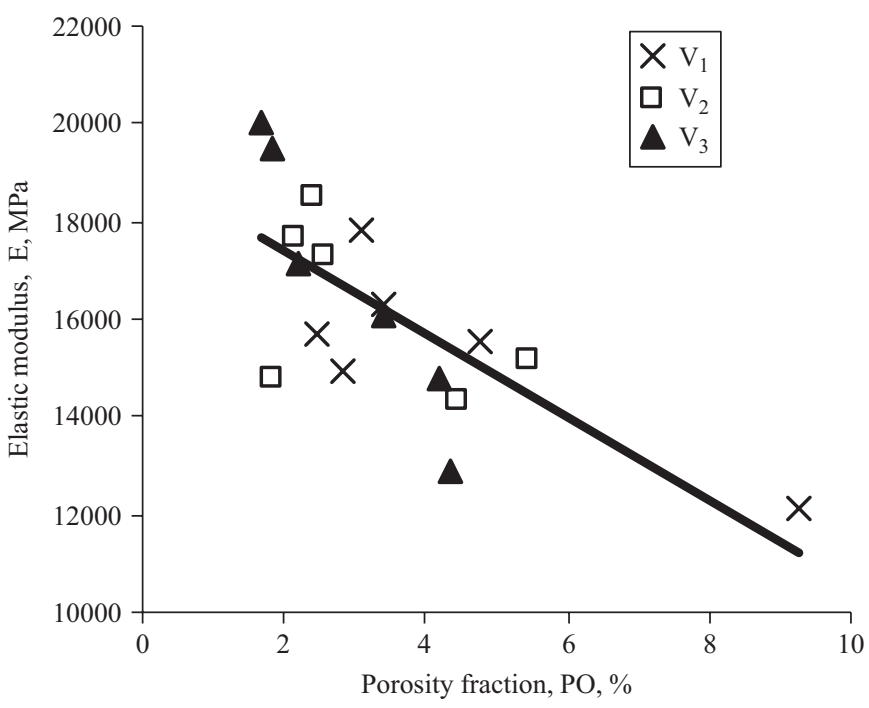

Fig. 5. Linear regression results of elastic modulus vs. porosity for the 18 specimens. Regression parameters are slope -842.7 , intercept 19,063 MPa, $p<0.001, R^{2}=0.54$.

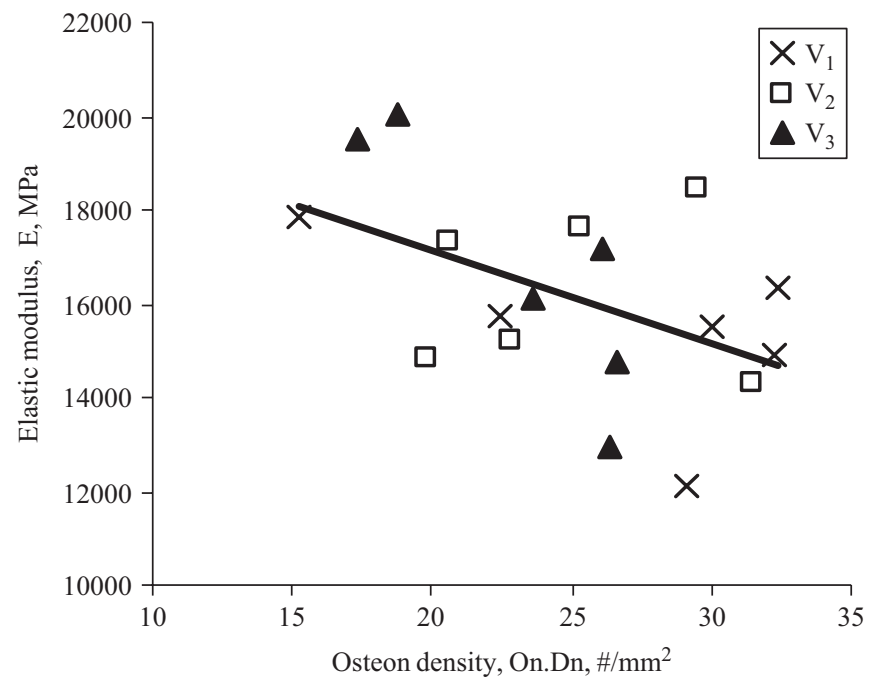

Fig. 6. Linear regression results of elastic modulus vs. osteon density for the 18 specimens. Regression parameters are slope -201.9 , intercept $21,192 \mathrm{MPa}, p=\theta .033, R^{2}=0.25$.

modulus $\left(p=\theta .327, R^{2}=\theta .06\right)$ or $\log \left(N_{\mathrm{f}}\right) \quad(p=\theta .282$, $\left.R^{2}=0.07\right)$.

\section{Discussion}

Stress fractures that occur in military recruits, athletes, and Thoroughbred racehorses are clinical manifestations of bone biology under the influence of the mechanical processes of fatigue and fracture. The mechanistic principles causing these manifestations are not fully understood. However, it appears that variations in collagen, mineral, and other microstructural components of bone play significant roles in these failure processes (Schaffler et al., 1987; Schaffler et al., 1995; Currey, 2004; Wang and Puram, 2004). Increases in mineral content 
are known to increase stiffness and decrease post-yield deformation, making the bone tissue fail in a manner similar to a brittle ceramic (Currey, 2004). Additionally, it is clear that microscopic mechanical damage in the form of microcracks and diffuse tissue damage occurs when bones are loaded to physiological strain levels (Schaffler et al., 1990; Boyce et al., 1998; Martin, 2003b; O'Brien et al., 2003). Taylor (1998) has suggested that the interaction of such defects may cause bone fatigue life to exhibit a volume effect. In the present investigation, uniaxial load control fatigue tests were conducted on waisted rectangular specimens from mid-diaphyseal equine cortical bone. The hypothesis that smaller volumes of cortical bone exhibit longer fatigue lives than similarly tested larger volumes was supported by the current study.

Mixed model repeated measures analysis of variance (ANOVA) on $\log \left(N_{\mathrm{f}}\right)$ initially suggested that increases in specimen volume may result in decreased fatigue life for similarly tested specimens. The repeated subject effect made the model more complex. With the elimination of specimens failing outside the gage region, the model was more sensitive to the horse-subject factor. Specifically, the statistical result depended on whether horse was treated as a fixed or random effect. However, the overall trend was decreasing fatigue life with increasing volume, in concert with the presence of a volume effect.

Regressions of failure probability on fatigue life for each volume group indicated that the data are consistent with a two-parameter Weibull distribution. Additionally, the similarity in Weibull moduli across the individual volume groups suggests that the bone material in all three groups failed in a similar manner (Cattell and Kibble, 2001). If different flaw populations were driving failure in each of the different volume groups, or different test parameters were being used, the individual volume groups would be expected to exhibit different slopes and different Weibull moduli. For example, consider a test conducted in load control with a lower initial peak strain. Similar to the movement along a stress-life curve, increases in, and variability of, fatigue life would be expected, i.e. increased $N_{0}$ and decreased $m_{\mathrm{f}}$, respectively. The only discriminable difference between test groups here was the specimen volume and surface area, factors reflected in the differences in the mean $\log \left(N_{\mathrm{f}}\right)$.

The regression between the logarithm of fatigue life and the logarithm of volume confirmed a significant volume effect on the fatigue life of equine cortical bone. The Weibull modulus or slope, derived from tests involving similar stress states, characterizes the variation in fatigue life of a particular material independent of its volume. The similarity of the Weibull volume modulus $\left(m_{\mathrm{V}}=4.47\right)$ to the individual moduli from the independent Weibull analyses $\left(m_{\mathrm{f} 1}=4.65, m_{\mathrm{f} 2}=4.62, m_{\mathrm{f} 3}=4.43\right)$ supports the hypothesis that these Weibull moduli are independent of volume. Under such conditions a definitive volume effect on fatigue life exists for the material (Wisnom, 1999; Cattell and Kibble, 2001).
We and others (Gibson et al., 1995; Martin et al., 1996; Gibeling et al., 2001; Skedros et al., 2003; Bigley et al., 2006) have shown that cortical bone mechanical properties exhibit anatomical regional differences attributable to variability in microstructural features. It has also been suggested that these microstructural features may be adapted to improve the mechanical performance of the cortical tissue (Gibson et al., 2006). The current histomorphometric analysis includes potentially relevant microstructural variables. We hypothesized that the volume effect would explain variability of fatigue life not accounted for by additional unspecified microstructural variables distributed throughout the bone tissue. We postulate that these microstructural features act as stress concentration sites for crack initiation and thus should result in decreased fatigue life, consistent with both Weibull theory and our data.

Application of Weibull theory to bone has limitations. In its power law form (Eq. (1)), no characteristic material volume is identifiable. A test specimen whose size approaches the dimensions of its inhomogeneities must have a characteristic volume (Bazant, 2005). For this reason Weibull theory is applicable only when these microstructures are sufficiently small compared with the test specimen size (Choi and Goldstein, 1992; Taylor et al., 1999; Bazant, 2005). In the present experiment, this criterion was met: the largest inhomogeneities were osteons and resorption cavities, having lateral dimensions of 163 and $108 \mu \mathrm{m}$ respectively, about $3-6 \%$ of the test specimens' thickness.

Additionally, Weibull theory describes brittle material failure in which a microscopic crack grows to macroscopic size. It does not account for local stress redistributions, microstructural barrier effects, or energy dissipation mechanisms associated with crack propagation in quasibrittle materials (Bazant, 2005). The incorporation of microstructural energy release, stress redistribution, barrier effects, and load sharing mechanisms requires more advanced size effect analyses (Bazant, 2005). Despite these limitations, Weibull theory was consistent with the data, supporting the existence of volume effects in cortical bone for specimens substantially larger than the material inhomogeneities.

The skeleton plays an essential role in the mechanical functioning and stability of the body. Among organ systems, bone and muscle tissue are the two largest components of total body weight. It has been suggested that vertebrate muscle mass is proportional to bone mass, and the mass and volume of these tissues are determinants of the metabolic costs of tissue maintenance and locomotion (Martin, 2003a; Daly et al., 2004). During daily activities, bones are repeatedly stressed and routinely accumulate damage. Bone damage is associated with the activation of remodeling responses that are the means for its removal (Mori and Burr, 1993; Martin, 2002). When the rates of damage accumulation exceed the rates of repair, catastrophic failure in the form of stress fractures can occur. 
Bone remodeling repairs damage, permitting a lighter skeleton. By accepting relatively high physiologic strains and repairing the resulting damage, the metabolic costs of a heavy skeleton are reduced (Martin, 2003a; Daly et al., 2004). The present results demonstrate that in addition to microstructural effects, one should also consider the specimen's volume as an explanatory variable with respect to fatigue life: for a given type of bone tissue smaller volumes of bone are more fatigue resistant than larger volumes, providing an additional advantage for a lighter, metabolically less-expensive skeleton.

\section{Conflict of interest}

None of these authors has conflicts of interest

\section{Acknowledgments}

This work was supported by the Doris Linn Chair of Bone Biology. The authors are grateful to Shane Curtiss, Justin Creel and Ron June for helpful discussions and technical assistance.

\section{References}

Bazant, Z.P., 2005. Scaling of Structural Strength. Elsevier, Burlington, MA, pp. 1-20, 53-76.

Bigley, R.F., Griffin, L.V., Christensen, L., Vandenbosch, R., 2006. Osteon interfacial strength and histomorphometry of equine cortical bone. Journal of Biomechanics 39, 1629-1640.

Boyce, T.M., Fyhrie, D.P., Glotkowski, M.C., Radin, E.L., Schaffler, M.B., 1998. Damage type and strain mode associations in human compact bone bending fatigue. Journal of Orthopaedic Research 16, 322-329.

Buckwalter, J.A., Glimcher, M.J., Cooper, R.R., Recker, R., 1995. Bone biology .1. Structure, blood-supply, cells, matrix, and mineralization. Journal of Bone and Joint Surgery-American 77A, 1256-1275.

Cattell, M.K., Kibble, K.A., 2001. Determination of the relationship between strength and test method for glass fibre epoxy composite coupons using Weibull analysis. Materials and Design 22, 245-250.

Choi, K., Goldstein, S.A., 1992. A comparison of the fatigue behavior of human trabecular and cortical bone tissue. Journal of Biomechanics $25,1371-1381$

Currey, J.D., 1964. Three analogies to explain the mechanical properties of bone. Biorheology 2, 1-10.

Currey, J.D., 2004. Tensile yield in compact bone is determined by strain, post-yield behaviour by mineral content. Journal of Biomechanics 37, 549-556.

Daly, R.M., Saxon, L., Turner, C.H., Robling, A.G., Bass, S.L., 2004. The relationship between muscle size and bone geometry during growth and in response to exercise. Bone 34, 281-287.

Gibeling, J.C., Shelton, D.R., Malik, C.L., 2001. Application of fracture mechanics to the study of crack propagation in bone. In: Rack, H., et al. (Eds.), Structural Biomaterials for the 21st Century. The Minerals, Metals and Materials Society, Warrendale, PA, pp. 239-254.

Gibson, V.A., Stover, S.M., Martin, R.B., Gibeling, J.C., Willits, N.H., Gustafson, M.B., Griffin, L.V., 1995. Fatigue behavior of the equine third metacarpus: mechanical property analysis. Journal of Orthopaedic Research 13, 861-868.

Gibson, V.A., Stover, S.M., Gibeling, J.C., Hazelwood, S.J., Martin, R.B., 2006. Osteonal effects on elastic modulus and fatigue life in equine bone. Journal of Biomechanics 39, 217-225.
Griffith, A.A., 1920. The phenomena of rupture and flow in solids. Philosophical Transactions of the Royal Society 221, 162-198.

Gustafson, M.B., Martin, R.B., Gibson, V., Storms, D.H., Stover, S.M., Gibeling, J., Griffin, L., 1996. Calcium buffering is required to maintain bone stiffness in saline solution. Journal of Biomechanics 29 , 1191-1194.

Hertzberg, R.W., 1996. Deformation and Fracture Mechanics of Engineering Materials. Wiley, New York, pp. 266-272.

Hogan, H.A., 1992. Micromechanics modeling of Haversian cortical bone properties. Journal of Biomechanics 25, 549-556.

Martin, R.B., 2002. Is all cortical bone remodeling initiated by microdamage? Bone 30, 8-13.

Martin, R.B., 2003a. Fatigue damage, remodeling, and the minimization of skeletal weight. Journal of Theoretical Biology 220, 271-276.

Martin, R.B., 2003b. Fatigue microdamage as an essential element of bone mechanics and biology. Calcified Tissue International 73, 101-107.

Martin, R.B., Gibson, V.A., Stover, S.M., Gibeling, J.C., Griffin, L.V., 1996. Osteonal structure in the equine third metacarpus. Bone 19 , $165-171$

Martin, R.B., Burr, D.B., Sharkey, N.A., 1998. Skeletal Tissue Mechanics. Springer, New York, pp. 29-30, 131-134, 143-151.

Mori, S., Burr, D.B., 1993. Increased intracortical remodeling following fatigue damage. Bone 14, 103-109.

Nunamaker, D.M., Butterweck, D.M., Provost, M.T., 1990. Fatigue fractures in Thoroughbred racehorses: relationships with age, peak bone strain, and training. Journal of Orthopaedic Research 8, 604-611.

O'Brien, F.J., Taylor, D., Lee, T.C., 2003. Microcrack accumulation at different intervals during fatigue testing of compact bone. Journal of Biomechanics 36, 973-980.

O'Brien, F.J., Taylor, D., Clive Lee, T., 2005. The effect of bone microstructure on the initiation and growth of microcracks. Journal of Orthopaedic Research 23, 475-480.

Rentzsch, W.H., 2003. A simple tool for designing with ceramics. Advanced Engineering Materials 5, 218-222.

Schaffler, M.B., Burr, D.B., Frederickson, R.G., 1987. Morphology of the osteonal cement line in human bone. Anatomical Record 217, 223-228

Schaffler, M.B., Radin, E.L., Burr, D.B., 1990. Long-term fatigue behavior of compact bone at low strain magnitude and rate. Bone $11,321-326$

Schaffler, M.B., Choi, K., Milgrom, C., 1995. Aging and matrix microdamage accumulation in human compact bone. Bone 17 , $521-525$.

Skedros, J.G., Dayton, M.R., Sybrowsky, C.L., Bloebaum, R.D., Bachus, K.N., 2003. Are uniform regional safety factors an objective of adaptive modeling/remodeling in cortical bone? Journal of Experimental Biology 206, 2431-2439.

Taylor, D., 1998. Fatigue of bone and bones: an analysis based on stressed volume. Journal of Orthopaedic Research 16, 163-169.

Taylor, D., Kuiper, J.H., 2001. The prediction of stress fractures using a "stressed volume" concept. Journal of Orthopaedic Research 19, 919-926.

Taylor, D., O'Brien, F., Prina-Mello, A., Ryan, C., O'Reilly, P., Lee, T.C., 1999. Compression data on bovine bone confirms that a "stressed volume" principle explains the variability of fatigue strength results. Journal of Biomechanics 32, 1199-1203.

Taylor, D., Casolari, E., Bignardi, C., 2004. Predicting stress fractures using a probabilistic model of damage, repair and adaptation. Journal of Orthopaedic Research 22, 487-494.

Wang, X., Puram, S., 2004. The toughness of cortical bone and its relationship with age. Annals of Biomedical Engineering 32, 123-135.

Weibull, W., 1951. A statistical distribution function of wide applicability. Journal of Applied Mechanics 18, 293-297.

Wisnom, M.R., 1999. Size effects in the testing of fibre-composite materials. Composites Science and Technology 59, 1937-1957.

Yeh, O. C., Martin, R. B., 2003. Volume effects in bone fatigue: Implications for bone adaptation and remodeling. In 50th Annual Meeting of Orthopaedic Research Scoiety. San Francisco. 